Pacific

Journal of

Mathematics

ON KRONECKER PRODUCTS OF SPIN CHARACTERS OF THE DOUBLE COVERS OF THE SYMMETRIC GROUPS

Christine Bessenrodt and Alexander S. Kleshchev

Volume $198 \quad$ No. 2

April 2001 


\title{
ON KRONECKER PRODUCTS OF SPIN CHARACTERS OF THE DOUBLE COVERS OF THE SYMMETRIC GROUPS
}

\author{
Christine Bessenrodt and Alexander S. Kleshchev
}

In this article, restrictions on the constituents of Kronecker products of spin characters of the double covers of the symmetric groups are derived. This is then used to classify homogeneous and irreducible products of spin characters; as an application of this, certain homogeneous 2-modular tensor products for the symmetric groups are described.

\section{Introduction.}

In recent years, a number of results on Kronecker products of complex $S_{n^{-}}$ characters have been obtained. In particular, the rectangular hull for the constituents in such products was found, and this was used for the classification of products with few homogeneous components; see [1] for this classification result and references to related work.

Here, we provide similar results for products of spin characters for the double covers $\widetilde{S}_{n}$ of the symmetric groups. The rectangular hull for spin products is determined in Theorem 3.2; this result serves as a crucial tool for the classification of homogeneous spin products in Theorem 4.2. (A module is called homogeneous if all of its composition factors are isomorphic to each other.) Finally, Theorem 4.2 is applied to prove a recent conjecture of Gow and Kleshchev describing certain homogeneous 2-modular tensor products for the symmetric groups (see Theorem 5.1).

\section{Preliminaries.}

We denote by $P(n)$ the set of partitions of $n$. For a partition $\lambda \in P(n)$, $l(\lambda)$ denotes its length, i.e., the number of (non-zero) parts of $\lambda$. The set of partitions of $n$ into odd parts only is denoted by $O(n)$, and the set of partitions of $n$ into distinct parts is denoted by $D(n)$. We write $D^{+}(n)$ (resp. $D^{-}(n)$ ) for the sets of partitions $\lambda$ in $D(n)$ with $n-l(\lambda)$ even (resp. odd); the partition $\lambda$ is then also called even (resp. odd).

We write $S_{n}$ for the symmetric group on $n$ letters, and $\widetilde{S}_{n}$ for one of its double covers; so $\widetilde{S}_{n}$ is a non-split extension of $S_{n}$ by a central subgroup $\langle z\rangle$ of order 2 . It is well-known that the representation theory of these 
double covers is 'the same' for all representation theoretical purposes. The spin characters of $\widetilde{S}_{n}$ are those that do not have $z$ in their kernel. For an introduction to the properties of spin characters (resp. for some results we will need in the sequel) we refer to $[\mathbf{6}],[\mathbf{1 0}],[\mathbf{1 1}],[\mathbf{1 3}]$. Below we collect some of the necessary notation and some results from $[\mathbf{1 3}]$ that are crucial in later sections.

For $\lambda \in P(n)$, we write $[\lambda]$ for the corresponding irreducible character of $S_{n}$; this is identified with the corresponding character of $\widetilde{S}_{n}$. The associate classes of spin characters of $\widetilde{S}_{n}$ are labelled canonically by the partitions in $D(n)$. For each $\lambda \in D^{+}(n)$ there is a self-associate spin character $\langle\lambda\rangle=$ $\operatorname{sgn}\langle\lambda\rangle$, and to each $\lambda \in D^{-}(n)$ there is a pair of associate spin characters $\langle\lambda\rangle,\langle\lambda\rangle^{\prime}=\operatorname{sgn}\langle\lambda\rangle$. We write

$$
\begin{aligned}
& \widehat{\langle\lambda\rangle}= \begin{cases}\langle\lambda\rangle & \text { if } \lambda \in D^{+}(n) \\
\langle\lambda\rangle+\langle\lambda\rangle^{\prime} & \text { if } \lambda \in D^{-}(n)\end{cases} \\
& \varepsilon_{\lambda}=\left\{\begin{array}{ll}
1 & \text { if } \lambda \in D^{+}(n) \\
\sqrt{2} & \text { if } \lambda \in D^{-}(n)
\end{array} .\right.
\end{aligned}
$$

In [13], Stembridge introduces a projective analogue of the outer tensor product, called the reduced Clifford product, and proves a shifted analogue of the LR rule which we will need in the sequel. To state this, we first have to define some further combinatorial notions.

Let $A^{\prime}$ be the ordered alphabet $\left\{1^{\prime}<1<2^{\prime}<2<\ldots\right\}$. The letters $1^{\prime}, 2^{\prime}, \ldots$ are said to be marked, the others are unmarked. The notation $|a|$ refers to the unmarked version of a letter $a$ in $A^{\prime}$. To a partition $\lambda \in D(n)$ we associate a shifted diagram

$$
Y^{\prime}(\lambda)=\left\{(i, j) \in \mathbb{N}^{2} \mid 1 \leq i \leq l(\lambda), i \leq j \leq \lambda_{i}+i-1\right\} .
$$

A shifted tableau $T$ of shape $\lambda$ is a map $T: Y^{\prime}(\lambda) \rightarrow A^{\prime}$ such that $T(i, j) \leq$ $T(i+1, j), T(i, j) \leq T(i, j+1)$ for all $i, j$ and the following additional property holds. Every $k \in\{1,2, \ldots\}$ appears at most once in each column of $T$, and every $k^{\prime} \in\left\{1^{\prime}, 2^{\prime}, \ldots\right\}$ appears at most once in each row of $T$. For $k \in\{1,2, \ldots\}$, let $c_{k}$ be the number of boxes $(i, j)$ in $Y^{\prime}(\lambda)$ such that $|T(i, j)|=k$. Then we say that the tableau $T$ has content $\left(c_{1}, c_{2}, \ldots\right)$. Analogously, we define skew shifted diagrams and skew shifted tableaux of skew shape $\lambda / \mu$ if $\mu$ is a partition with $Y^{\prime}(\mu) \subseteq Y^{\prime}(\lambda)$. For a (possibly skew) shifted tableau $S$ we define its associated word $w(S)=w_{1} w_{2} \cdots$ by reading the rows of $S$ from left to right and from bottom to top. By erasing the marks of $w$, we obtain the word $|w|$.

Given a word $w=w_{1} w_{2} \ldots$, we define

$$
\begin{aligned}
m_{i}(j) & =\text { multiplicity of } i \text { among } w_{n-j+1}, \ldots, w_{n} & & (\text { for } 0 \leq j \leq n), \\
m_{i}(n+j) & =m_{i}(n)+\text { multiplicity of } i^{\prime} \text { among } w_{1}, \ldots, w_{j} & & (\text { for } 0<j \leq n) .
\end{aligned}
$$


This function $m_{i}$ corresponds to reading the rows of the tableau first from right to left and from top to bottom, counting the letter $i$ on the way, and then reading from bottom to top and left to right, counting the letter $i^{\prime}$ on this way.

The word $w$ satisfies the lattice property if, whenever $m_{i}(j)=m_{i-1}(j)$, then

$$
\begin{aligned}
w_{n-j} & \neq i, i^{\prime}, & & \text { if } 0 \leq j<n, \\
w_{j-n+1} & \neq i-1, i^{\prime}, & & \text { if } n \leq j<2 n .
\end{aligned}
$$

For two partitions $\mu$ and $\nu$ we denote by $\mu \cup \nu$ the partition which has as its parts all the parts of $\mu$ and $\nu$ together.

Theorem 2.1 ([13, 8.1 and 8.3]). Let $\mu \in D(k), \nu \in D(n-k), \lambda \in D(n)$, and form the reduced Clifford product $\langle\mu\rangle \times_{c}\langle\nu\rangle$. Then we have

$$
\left(\left(\langle\mu\rangle \times_{c}\langle\nu\rangle\right) \uparrow \widetilde{S}_{n},\langle\lambda\rangle\right)=\frac{1}{\varepsilon_{\lambda} \varepsilon_{\mu \cup \nu}} 2^{(l(\mu)+l(\nu)-l(\lambda)) / 2} f_{\mu \nu}^{\lambda},
$$

unless $\lambda$ is odd and $\lambda=\mu \cup \nu$. In that latter case, the multiplicity of $\langle\lambda\rangle$ is 0 or 1 , according to the choice of associates.

The coefficient $f_{\mu \nu}^{\lambda}$ is the number of shifted tableaux $S$ of shape $\lambda / \mu$ and content $\nu$ such that the tableau word $w=w(S)$ satisfies the lattice property and the leftmost $i$ of $|w|$ is unmarked in $w$ for $1 \leq i \leq l(\nu)$.

We will also use the following result from [13] on inner tensor products with the basic spin character $\langle n\rangle$ :

Theorem $2.2([\mathbf{1 3}, 9.3])$. Let $\lambda \in D(n), \mu$ a partition of $n$. We have

$$
(\langle n\rangle[\mu],\langle\lambda\rangle)=\frac{1}{\varepsilon_{\lambda} \varepsilon_{(n)}} 2^{(l(\lambda)-1) / 2} g_{\lambda \mu},
$$

unless $\lambda=(n), n$ is even, and $\mu$ is a hook partition. In that case, the multiplicity of $\langle\lambda\rangle$ is 0 or 1 according to choice of associates.

The coefficient $g_{\lambda \mu}$ is the number of "shifted tableaux" $S$ of unshifted shape $\mu$ and content $\lambda$ such that the tableau word $w=w(S)$ satisfies the lattice property and the leftmost $i$ of $|w|$ is unmarked in $w$ for $1 \leq i \leq l(\lambda)$.

\section{On bounds for the constituents of spin products.}

First we prove a spin version of a result of Dvir [4] (resp. Clausen and Meier $[3])$, describing the rectangular hull of the constituents in the Kronecker product of two spin characters.

Definition 3.1. Let $\mu, \nu \in D(n)$. Define the coefficients $d_{\mu \nu}^{\lambda}, \lambda \in P(n)$, by

$$
\langle\mu\rangle \cdot\langle\nu\rangle=\sum_{\lambda \vdash n} d_{\mu \nu}^{\lambda}[\lambda] .
$$


Then define the width $w$ and the height $h$ of the product by

$$
\begin{aligned}
& w(\langle\mu\rangle \cdot\langle\nu\rangle)=\max \left\{\lambda_{1} \mid \exists \lambda=\left(\lambda_{1}, \ldots\right): d_{\mu \nu}^{\lambda} \neq 0\right\} \\
& h(\langle\mu\rangle \cdot\langle\nu\rangle)=\max \left\{l \mid \exists \lambda=\left(\lambda_{1}, \ldots, \lambda_{l}>0\right): d_{\mu \nu}^{\lambda} \neq 0\right\} .
\end{aligned}
$$

The rectangular partition $\left(w^{h}\right)$ is then called the rectangular hull $R(\langle\mu\rangle \cdot\langle\nu\rangle)$ of the product $\langle\mu\rangle \cdot\langle\nu\rangle$.

Remark. Since $\langle\mu\rangle^{\prime}=\operatorname{sgn} \cdot\langle\mu\rangle$, we can easily obtain all products of spin characters if we know the coefficients $d_{\mu \nu}^{\lambda}$ above.

Theorem 3.2. Let $\mu, \nu \in D(n)$.

Then the rectangular hull of the product $\langle\mu\rangle \cdot\langle\nu\rangle$ is given by

$$
R(\langle\mu\rangle \cdot\langle\nu\rangle)=\left(|\mu \cap \nu|^{|\mu \cap \nu|}\right),
$$

except in the case when $\mu=\nu \in D^{-}(n)$.

In the case $\mu=\nu \in D^{-}(n)$, but $\mu$ not a staircase partition $(k, k-$ $1, \ldots, 2,1)$, we have

$$
R(\langle\mu\rangle \cdot\langle\mu\rangle)=\left\{\begin{array}{ll}
\left((n-1)^{n}\right) & \text { if } n-l(\mu) \equiv 1 \bmod 4 \\
\left(n^{n-1}\right) & \text { if } n-l(\mu) \equiv 3 \bmod 4
\end{array} .\right.
$$

More precisely,

$$
\left|\left\{j \mid \mu_{j}>\mu_{j+1}+1\right\}\right|=\left\{\begin{array}{ll}
(\langle\mu\rangle \cdot\langle\mu\rangle,[n-1,1]) & \text { if } n-l(\mu) \equiv 1 \bmod 4 \\
\left(\langle\mu\rangle \cdot\langle\mu\rangle,\left[2,1^{n-2}\right]\right) & \text { if } n-l(\mu) \equiv 3 \bmod 4
\end{array} .\right.
$$

Finally, if $n=\left(\begin{array}{c}k+1 \\ 2\end{array}\right)$ is a triangular number with $k \equiv 2$ or $3 \bmod 4$, and $\mu=\nu=(k, k-1, \ldots, 2,1) \in D^{-}(n)$, then we have

$$
R(\langle\mu\rangle \cdot\langle\mu\rangle)=\left\{\begin{array}{ll}
\left((n-2)^{n}\right) & \text { if } k \equiv 2 \text { or } 7 \bmod 8 \\
\left(n^{n-2}\right) & \text { if } k \equiv 3 \text { or } 6 \bmod 8
\end{array} .\right.
$$

More precisely,

$$
1=\left\{\begin{array}{ll}
\left(\langle\mu\rangle \cdot\langle\mu\rangle,\left[n-2,1^{2}\right]\right) & \text { if } k \equiv 2 \text { or } 7 \bmod 8 \\
\left(\langle\mu\rangle \cdot\langle\mu\rangle,\left[3,1^{n-3}\right]\right) & \text { if } k \equiv 3 \text { or } 6 \bmod 8
\end{array} .\right.
$$

Remark. The number $\left|\left\{j \mid \mu_{j}>\mu_{j+1}+1\right\}\right|$ is almost the number of boxes $A$ that can be removed such that the resulting partition $\mu \backslash A$ is in $D(n-1)$; the only exception is that a final part 1 is not counted. This is the reason for the further exception in the case of staircase partitions.

Proof. Let $\lambda=\left(\lambda_{1}, \lambda_{2}, \ldots\right)$ be a partition of $n$ with $(\langle\mu\rangle \cdot\langle\nu\rangle,[\lambda]) \neq 0$. Put $k=\lambda_{1}$. Then $\left([k],[\lambda] \downarrow_{\widetilde{S}_{k}}\right) \neq 0$, and hence

$$
\begin{aligned}
0 \neq\left(\langle\mu\rangle \downarrow_{\widetilde{S}_{k}} \cdot\langle\nu\rangle \downarrow_{\widetilde{S}_{k}},[k]\right) & =\left(\langle\nu\rangle \downarrow_{\widetilde{S}_{k}}, \overline{\langle\mu\rangle} \downarrow_{\widetilde{S}_{k}}\right) \\
& = \begin{cases}\left(\langle\nu\rangle \downarrow_{\widetilde{S}_{k}},\langle\mu\rangle^{\prime} \downarrow_{\widetilde{S}_{k}}\right), & \text { if } n-l(\mu) \equiv 1 \bmod 4 \\
\left(\langle\nu\rangle \downarrow_{\widetilde{S}_{k}},\langle\mu\rangle \downarrow_{\widetilde{S}_{k}}\right), & \text { else. }\end{cases}
\end{aligned}
$$


Indeed, the last equation follows since clearly $\overline{\langle\rho\rangle}=\langle\rho\rangle$ for each $\rho \in D^{+}(n)$, and for $\rho \in D^{-}(n)$ the only possibly non-real values can occur at classes of cycle type $\rho$, where $\langle\rho\rangle$ takes value $i^{(n-l(\rho)+1) / 2} \sqrt{\frac{\prod_{j} \rho_{j}}{2}}$ (see [6], [10]). Hence $\overline{\langle\rho\rangle}=\langle\rho\rangle$ if $n-l(\rho) \equiv 3 \bmod 4$, and $\overline{\langle\rho\rangle}=\langle\rho\rangle^{\prime}$ if $n-l(\rho) \equiv 1 \bmod 4$. In any case, there exists $\alpha \in D(k)$ such that $\alpha \subseteq \mu \cap \nu$, so $k \leq|\mu \cap \nu|$.

Multiplying with the sign character, the same argument also gives the inequality $l(\lambda) \leq|\mu \cap \nu|$.

Put $m:=|\mu \cap \nu|$. The character $\langle\mu \cap \nu\rangle$ or its associate is a common constituent of $\langle\mu\rangle \downarrow_{\widetilde{S}_{m}}$ and $\langle\nu\rangle \downarrow_{\widetilde{S}_{m}}$ (and their associates), unless $\mu=\nu \in$ $D^{-}(n)$, when $\left(\langle\mu\rangle,\langle\mu\rangle^{\prime}\right)=0$. Hence, if we are not in the exceptional case, then by the argument above

$$
0 \neq\left(\langle\mu\rangle \downarrow_{\widetilde{S}_{m}} \cdot\langle\nu\rangle \downarrow_{\widetilde{S}_{m}},[m]\right) .
$$

Hence there must be a constituent $[\lambda]$ in the product with first part $\lambda_{1} \geq m$, and by what we have already proved, we have in fact equality $\lambda_{1}=m$. This argument is independent of the choice of associates so we also obtain $h(\langle\mu\rangle \cdot\langle\nu\rangle)=m$ unless we are in the exceptional case.

We now have to deal with the case that $\mu=\nu \in D^{-}(n)$. If $n-l(\mu) \equiv$ $1 \bmod 4$, then $\overline{\langle\mu\rangle}=\langle\mu\rangle^{\prime}$, as we have already noted above, so $(\langle\mu\rangle \cdot\langle\mu\rangle,[n])=$ 0 . Hence

$$
\begin{aligned}
(\langle\mu\rangle \cdot\langle\mu\rangle,[n-1,1]) & =\left(\langle\mu\rangle \cdot\langle\mu\rangle,[n-1] \uparrow \widetilde{S}_{n}\right) \\
& =\left(\langle\mu\rangle \downarrow \widetilde{S}_{n-1} \cdot\langle\mu\rangle \downarrow \widetilde{S}_{n-1},[n-1]\right) \\
& =\left(\langle\mu\rangle \downarrow \widetilde{S}_{n-1},\langle\mu\rangle^{\prime} \downarrow_{\widetilde{S}_{n-1}}\right) \\
& =\left|\left\{j \mid \mu_{j}>\mu_{j+1}+1\right\}\right|,
\end{aligned}
$$

where the last equality follows from the spin branching rule. In particular, if $\mu$ is not the staircase $(k, k-1, \ldots, 1)$, then $w(\langle\mu\rangle \cdot\langle\mu\rangle)=n-1$. Since

$$
\left(\langle\mu\rangle \cdot\langle\mu\rangle,\left[1^{n}\right]\right)=(\langle\mu\rangle,\langle\mu\rangle)=1,
$$

the assertion on the height follows immediately.

If $n-l(\mu) \equiv 3 \bmod 4$, then $\overline{\langle\mu\rangle}=\langle\mu\rangle$. By a similar reasoning as above we obtain the assertion in this case except for the height statement in the case of a staircase partition.

Finally, we have to deal with the case where $\mu=(k, k-1, \ldots, 1) \in D^{-}(n)$. Here,

$$
n-l(\mu) \equiv\left\{\begin{array}{ll}
1 \bmod 4 & \text { if } k \equiv 2 \text { or } 7 \bmod 8 \\
3 \bmod 4 & \text { if } k \equiv 3 \text { or } 6 \bmod 8
\end{array} .\right.
$$

We consider the case where $k \equiv 2$ or $7 \bmod 8$; the other case is dual. As $n-l(\mu) \equiv 1 \bmod 4$, we already know from the previous arguments that 
$w(\langle\mu\rangle \cdot\langle\mu\rangle) \leq n-2$. Hence

$$
\begin{aligned}
& (\langle\mu\rangle \cdot\langle\mu\rangle,[n-2,2]) \\
& =\left(\langle\mu\rangle \downarrow_{\widetilde{S}_{n-2,2}},\langle\mu\rangle^{\prime} \downarrow_{\widetilde{S}_{n-2,2}}\right) \\
& =\left(\langle k, k-1, \ldots, 3,1\rangle \times_{c}\langle 2\rangle,\langle k, k-1, \ldots, 3,1\rangle \times_{c}\langle 2\rangle^{\prime}\right) \\
& =0,
\end{aligned}
$$

where the restriction to $\widetilde{S}_{n-2,2}$ follows from Theorem 2.1. Thus, using the spin branching rule we obtain

$$
\begin{aligned}
\left(\langle\mu\rangle \cdot\langle\mu\rangle,\left[n-2,1^{2}\right]\right) & =\left(\langle\mu\rangle \downarrow_{\widetilde{S}_{n-2,1^{2}}},\langle\mu\rangle^{\prime} \downarrow_{\widetilde{S}_{n-2,1^{2}}}\right) \\
& =(\langle k, k-1, \ldots, 3,1\rangle,\langle k, k-1, \ldots, 3,1\rangle) \\
& =1 .
\end{aligned}
$$

Hence $R(\langle\mu\rangle \cdot\langle\mu\rangle)=\left((n-2)^{n}\right)$ in this case, as claimed.

For making the previous result slightly more precise, we need spin versions of some results in $[\mathbf{1}]$ :

Lemma 3.3. Let $a_{1}, a_{2}, b_{1}, b_{2} \in \mathbb{N}_{0}$ with $a_{1}>a_{2}, b_{1}>b_{2}, a_{1}+a_{2} \geq b_{1}+b_{2}$. Then

$$
b_{1}+b_{2}=\min \left(a_{1}+a_{2}, b_{1}+b_{2}\right) \leq 2\left(\min \left(a_{1}, b_{1}\right)+\min \left(a_{2}, b_{2}\right)\right)-1,
$$

and equality holds if and only if $\left(a_{1}, a_{2}, b_{1}, b_{2}\right)$ is of the form $\left(a_{1}, 0, b_{1}, b_{1}-1\right)$ or $\left(a_{1}, a_{2}, 1,0\right)$ or $\left(a_{1}, a_{1}-1,2 a_{1}-1,0\right)$.

Proof. First we consider the case when $b_{1} \leq a_{1}$. Then the right hand side is

$$
2\left(b_{1}+\min \left(a_{2}, b_{2}\right)\right)-1 \geq 2 b_{1}-1 \geq b_{1}+b_{2},
$$

and equality holds if and only if $\min \left(a_{2}, b_{2}\right)=0$ and $b_{1}=b_{2}+1$. These are the cases where $\left(a_{1}, a_{2}, b_{1}, b_{2}\right)$ is of the form $\left(a_{1}, 0, b_{1}, b_{1}-1\right)$ or $\left(a_{1}, a_{2}, 1,0\right)$.

In the case when $a_{1}<b_{1}$, we have $b_{2}<a_{2}<a_{1}<b_{1}$. Hence the right hand side is

$$
2\left(a_{1}+b_{2}\right)-1=\left(2 a_{1}-1\right)+2 b_{2} \geq a_{1}+a_{2}+2 b_{2} \geq b_{1}+3 b_{2} \geq b_{1}+b_{2},
$$

and equality holds if and only if $a_{2}=a_{1}-1, b_{1}+b_{2}=a_{1}+a_{2}$ and $b_{2}=0$. This is exactly the third situation described in the statement of the lemma.

We denote by $b_{i j}(\mu)$ the $(i, j)$-bar length of $\mu$; in particular, $b_{11}(\mu)=$ $\mu_{1}+\mu_{2}$ for $\mu=\left(\mu_{1}, \mu_{2}, \ldots\right)$. The partition $\mu$ has an $\ell$-bar if $b_{i j}(\mu)=\ell$ for some $(i, j)$. For details on the combinatorics of bars we refer to [12].

Lemma 3.4. Let $\mu, \nu \in D(n)$. Then

$$
\min \left(b_{11}(\mu), b_{11}(\nu)\right) \leq 2|\mu \cap \nu|-1,
$$

and equality holds if and only if one of $\mu, \nu$ is $(n)$ and the other one has first two parts $k, k-1$. 
Proof. Let $\mu=\left(\mu_{1}, \mu_{2}, \ldots\right), \nu=\left(\nu_{1}, \nu_{2}, \ldots\right)$. Then we have $b_{11}(\mu)=$ $\mu_{1}+\mu_{2}$ and $b_{11}(\nu)=\nu_{1}+\nu_{2}$. Furthermore, $|\mu \cap \nu|=\sum_{j} \min \left(\mu_{j}, \nu_{j}\right) \geq$ $\min \left(\mu_{1}, \nu_{1}\right)+\min \left(\mu_{2}, \nu_{2}\right)$. By the previous Lemma, applied to $\left(a_{1}, a_{2}, b_{1}, b_{2}\right)$ $=\left(\mu_{1}, \mu_{2}, \nu_{1}, \nu_{2}\right)$, we immediately obtain the assertion.

We denote by $h_{i j}(\lambda)$ the $(i, j)$-hook length of $\lambda=\left(\lambda_{1}, \ldots, \lambda_{l}\right)$; in particular, $h_{11}(\lambda)=\lambda_{1}+l-1$.

Theorem 3.5. Let $n \geq 4, \mu, \nu \in D(n)$, and assume we are not in the case that one of $\mu, \nu$ is $(n)$ and the other one has first two parts $k, k-1$. Let $\lambda$ be a partition such that $[\lambda]$ is a constituent of $\langle\mu\rangle \cdot\langle\nu\rangle$. Then

$$
h_{11}(\lambda)<2|\mu \cap \nu|-1 \text {. }
$$

Proof. Set $l=2|\mu \cap \nu|-1$. Take $\widetilde{\pi} \in \widetilde{S}_{n}$ of type $\left(l, 1^{n-l}\right) \in O(n), \pi$ the corresponding element in $S_{n}$. By Morris' recursion formula [11] we have:

$$
\langle\mu\rangle \cdot\langle\nu\rangle(\widetilde{\pi})=0
$$

since either $\mu$ or $\nu$ does not have an $l$-bar, by Lemma 3.4. By Theorem 3.2, for any constituent $[\lambda]$ of $\langle\mu\rangle \cdot\langle\nu\rangle$ we have $\lambda_{1} \leq|\mu \cap \nu|$ and $l(\lambda) \leq|\mu \cap \nu|$, so $h_{11}(\lambda) \leq 2|\mu \cap \nu|-1$. Thus $\lambda$ has an $l$-hook if and only if $\lambda_{1}=|\mu \cap \nu|=l(\lambda)$, which is then the hook $H_{11}=H_{11}(\lambda)$ of leg length $|\mu \cap \nu|-1$. So assume now that $[\lambda]$ is a constituent which has an l-hook; then by the MurnaghanNakayama formula [8]:

$$
[\lambda](\widetilde{\pi})=[\lambda](\pi)=(-1)^{|\mu \cap \nu|-1}\left[\lambda \backslash H_{11}\right](1) \neq 0 .
$$

So all such constituents $[\lambda]$ contribute a summand of the same sign to $\langle\mu\rangle$. $\langle\nu\rangle(\tilde{\pi})$. Since this latter value is zero, there can be no such constituent, and hence $h_{11}(\lambda)<2|\mu \cap \nu|-1$ for all constituents of $\langle\mu\rangle \cdot\langle\nu\rangle$.

Corollary 3.6. Let $n \geq 4, \mu, \nu \in D(n)$, and assume we are not in the situation that one of $\mu, \nu$ is $(n)$ and the other one has first two parts $k, k-1$. Then $\langle\mu\rangle \cdot\langle\nu\rangle$ has at least two different constituents.

Proof. Clearly, if $\mu \in D^{-}(n)$, then $\langle\mu\rangle \cdot\langle\mu\rangle$ contains one of $[n]$ or $\left[1^{n}\right]$ and at least one further constituent different from these. So we may assume now that we are not in the exceptional case of Theorem 3.2. Then the assertion follows from the inequality in Theorem 3.5 as the $(1,1)$-hook of the rectangle $\left(|\mu \cap \nu|^{|\mu \cap \nu|}\right)$ has length $2|\mu \cap \nu|-1$.

\section{Homogeneous spin character products.}

We start by proving the following combinatorial lemma.

Lemma 4.1. Let $k \in \mathbb{N}, n=k(k+1) / 2$, and let $\lambda=(k, k-1, \ldots, 2,1)$ be the staircase partition of height $k$. Let $H(k)$ be the product of the hook lengths in $\lambda$, and let $B(k)$ be the product of the bar lengths in $\lambda$. Then

$$
2^{n-k} H(k)=B(k) \text {. }
$$


Proof. We prove the claim by induction on $k$. For $k=1$ the assertion is clear. Now assume that $k \geq 2$ and that the assertion holds for $k-1$, i.e.,

$$
2^{(n-k)-(k-1)} H(k-1)=B(k-1) .
$$

When the staircase diagram of $(k-1, k-2, \ldots, 2,1)$ is extended by the first row $k$, it is clear how the products of hook (resp. bar) lengths change:

$$
H(k)=H(k-1) \cdot \prod_{i=1}^{k}(2 i-1), B(k)=B(k-1) \cdot \prod_{j=0}^{k-1}(k+j) .
$$

As

$$
\begin{aligned}
\prod_{j=0}^{k-1}(k+j)=\frac{(2 k-1) !}{(k-1) !} & =\frac{(1 \cdot 3 \cdots(2 k-1))(2 \cdot 4 \cdots 2(k-1))}{(k-1) !} \\
& =1 \cdot 3 \cdots(2 k-1) \cdot 2^{k-1}
\end{aligned}
$$

the result now follows immediately.

Theorem 4.2. Let $n \geq 4, \mu, \nu \in D(n)$. Then $\langle\mu\rangle \cdot\langle\nu\rangle$ is homogeneous if and only if $n$ is a triangular number, say $n=\left(\begin{array}{c}k+1 \\ 2\end{array}\right)$, one of $\mu, \nu$ is $(n)$ and the other one is $(k, k-1, \ldots, 2,1)$. In this case, we have

$$
\langle n\rangle \cdot\langle k, k-1, \ldots, 2,1\rangle=2^{a(k)}[k, k-1, \ldots, 2,1]
$$

where

$$
a(k)=\left\{\begin{array}{ll}
\frac{k-2}{\frac{2}{2}} & \text { if } k \text { is even } \\
\frac{k-3}{2} & \text { if } k \equiv 1 \bmod 4 .
\end{array} .\right.
$$

In particular, $\langle\mu\rangle \cdot\langle\nu\rangle$ is irreducible if and only if $n=6$ and the product is $\langle 6\rangle \cdot\langle 3,2,1\rangle=[3,2,1]$.

Proof. By Corollary 3.6 we only have to deal with the case $\mu=(n)$ and $\nu=(k, k-1, \ldots)$ for some $k$, where we have to show that the product is homogeneous if and only if $\nu$ is a staircase partition $(k, k-1, k-2, \ldots, 2,1)$.

First, we assume that the product $\langle n\rangle \cdot\langle\nu\rangle$, with $\nu=(k, k-1, \ldots)$, is homogeneous. In this case, we use the information given by Stembridge on products with the basic spin representation $\langle n\rangle$, i.e., Theorem 2.2. By this result, clearly $[\nu]$ is a constituent of $\langle n\rangle \cdot\langle\nu\rangle$. On the other hand, by Theorem 3.2 we know that there is a constituent $[\lambda]$ in the product with $l(\lambda)=k=|(n) \cap \nu|$. Since the product is assumed to be homogeneous, we must have $\nu=\lambda$, but as $\nu_{1}=k$ and $\nu \in D(n)$, we can only have $l(\nu)=k$ if $\nu=(k, k-1, \ldots, 2,1)$.

Finally, we have to prove the assertion on the products of the special form $\langle n\rangle \cdot\langle k, k-1, \ldots, 2,1\rangle$. As we have remarked above, by Theorem 2.2 we 
know that $[\nu]$ appears in the product $\langle n\rangle \cdot\langle\nu\rangle$, and for $\nu=(k, k-1, \ldots, 2,1)$ the multiplicity is

$$
\frac{1}{\varepsilon_{\nu} \varepsilon_{(n)}} 2^{(l(\nu)-1) / 2} g_{\nu \nu}=\frac{1}{\varepsilon_{\nu} \varepsilon_{(n)}} 2^{(k-1) / 2} .
$$

One easily checks that this equals $2^{a(k)}$, with $a(k)$ as given in the statement of the theorem.

To show that no other constituent occurs, we just check dimensions on both sides. Let $B(k)$ be the product of the bar lengths in $\nu=(k, k-$ $1, \ldots, 2,1)$, and let $H(k)$ be the product of the hook lengths in $\nu$. Then by the hook formula (resp. the bar formula) for the character degrees we have to show that

$$
2^{\left[\frac{n-1}{2}\right]} \cdot 2^{\left[\frac{n-k}{2}\right]} \frac{n !}{B(k)}=2^{a(k)} \frac{n !}{H(k)}
$$

with $a(k)$ as in the statement of the theorem. Considering the different cases depending on $k \bmod 4$, this is easily seen to be equivalent to

$$
2^{n-k} H(k)=B(k) \text {. }
$$

Hence the assertion follows from Lemma 4.1.

Remark 4.3. A completely different proof of Theorem 4.2 is based on the observation that the product of two non-associate spin characters gives an ordinary character of $S_{n}$ which vanishes on all 2-elements, and is thus the character of a projective module at characteristic 2 . Hence, if the product is homogeneous, this character is a multiple of an irreducible projective character of $S_{n}$; these irreducible characters are exactly the ones labelled by 2 -cores, i.e., staircase partitions. So the right hand side in Theorem 4.2 comes as no surprise.

\section{Application to 2-modular tensor products.}

In this section we want to apply the result about spin products for a proof of a recent conjecture by Gow and Kleshchev [5] describing certain homogeneous 2-modular tensor products for the symmetric groups. The argument for this proof was developed jointly with R. Gow.

Let $F$ be a field of characteristic 2 . We denote the irreducible $F S_{n}$-module labelled by the partition $\lambda \in D(n)$ by $D^{\lambda}$. We define the spin module $S$ to be the irreducible $F S_{n}$-module labelled by $(m+1, m-1)$ if $n=2 m$, and by $(m, m-1)$ if $n=2 m-1$.

Theorem 5.1. Let $n=\left(\begin{array}{c}k+1 \\ 2\end{array}\right)$ be a triangular number, and set $\mu=(k, k-$ $1, \ldots, 2,1)$ and $\lambda=(2 k-1,2 k-5,2 k-9, \ldots) \in D(n)$. Then

$$
S \otimes D^{\lambda} \simeq 2^{[(k-1) / 4]} D^{\mu} .
$$


Proof. By Theorem 4.2 we have

$$
\langle n\rangle \cdot\langle k, k-1, \ldots, 2,1\rangle=2^{a(k)}[k, k-1, \ldots, 2,1]
$$

with $a(k)$ described explicitly in Theorem 4.2. We know that the Brauer character $\phi$ of the reduction modulo 2 of the basic spin character $\langle n\rangle$ is irreducible, and it is the Brauer character for the spin module $S$. Furthermore, $\mu=(k, k-1, \ldots, 2,1)$ is a 2 -core, so the corresponding Brauer character $\chi$ of $[\mu] \bmod 2$ is also irreducible. Let $\psi$ be the Brauer character of the reduction $\bmod 2$ of $\langle\mu\rangle$.

By the formula above we have

$$
\phi \cdot \psi=2^{a(k)} \chi .
$$

Suppose that $\psi$ contains two different irreducible Brauer characters $\alpha$ and $\beta$. Then $\phi \alpha=s \chi$ and $\phi \beta=t \chi$ for certain positive integers $s$ and $t$. But the basic spin character $\langle n\rangle$ and hence also $\phi$ is non-zero on every 2-regular element and therefore $\alpha(g)=s \chi(g) / \phi(g)$ and $\beta(g)=t \chi(g) / \phi(g)$ holds for all 2-regular elements $g \in \widetilde{S}_{n}$. This implies that $\alpha$ and $\beta$ are linearly dependent, contradicting the assumption that they are two different irreducible Brauer characters.

Hence $\psi$ is a multiple of an irreducible Brauer character. For obtaining the precise decomposition of $\psi$ we use some results on the 2-decomposition matrix for $\widetilde{S}_{n}$ in [2]. First we have to introduce some notation. For a partition $\alpha \in D(n)$ let $\mathrm{dbl}^{2}(\alpha)$ be the 2-regular partition obtained as follows. First "double" $\alpha$ by breaking each part into two halves, i.e., an odd part $2 t-1$ is replaced by $t, t-1$, and an even part $2 t$ is replaced by $t, t$. Let $\beta$ be the resulting partition. Then we regularize $\beta$ into the 2 -regular partition $\beta^{R}=$ : $\operatorname{dbl}^{2}(\alpha)$ by pushing up nodes along the diagonal ladders of the 2 residue diagram (we refer the reader to [8], p. 282 for more details on this process). Now by [2], Theorem (5.2) we know that the final 2-decomposition number for each spin character $\langle\alpha\rangle$ occurs in the column labelled by $\mathrm{dbl}^{2}(\alpha)$, and this entry is precisely $2^{[m(\alpha) / 2]}$, where $m(\alpha)$ is the number of even parts of $\alpha$.

We apply this to $\mu=(k, k-1, \ldots, 2,1)$. It is easy to check that $\mathrm{dbl}^{2}(\mu)=$ $\lambda$. Keeping in mind that the Brauer character $\psi$ corresponding to $\mu$ is a multiple of an irreducible Brauer character, we thus obtain

$$
\psi=2^{[m(\mu) / 2]} \theta
$$

where $\theta$ is the irreducible Brauer character labelled by $\lambda$.

As $m(\mu)=[k / 2]$ we have thus shown that

$$
2^{[[k / 2] / 2]} \phi \cdot \theta=2^{a(k)} \chi .
$$

Using the explicit description of $a(k)$, we then obtain

$$
\phi \cdot \theta=2^{[(k-1) / 4]} \chi .
$$


This is equivalent to the assertion on the tensor product.

Acknowledgements. The financial support by the Deutsche Forschungsgemeinschaft (grants Be 923/6-1 and JAP-115/169/0) and the NSF (grants DMS-9600124 and DMS-9900134) is gratefully acknowledged. The first author would like to express her thanks to Professor Uno for the hospitality enjoyed at the University of Osaka where some work for this article was done. We would also like to thank R. Gow for the exchange of ideas on the proof of Theorem 5.1.

\section{References}

[1] C. Bessenrodt and A. Kleshchev, On Kronecker products of complex representations of the symmetric groups, Pacific J. Math., 190 (1999), 201-223.

[2] C. Bessenrodt and J. Olsson, The 2-blocks of the covering groups of the symmetric groups, Advances in Math., 129 (1997), 261-300.

[3] M. Clausen and H. Meier, Extreme irreduzible Konstituenten in Tensordarstellungen symmetrischer Gruppen, Bayreuther Math. Schriften, 45 (1993), 1-17.

[4] Y. Dvir, On the Kronecker product of $S_{n}$ characters, J. Algebra, 154 (1993), 125-140.

[5] R. Gow and A. Kleshchev, Connections between the representations of the symmetric group and the symplectic group in characteristic 2, J. Algebra, 221 (1999), 60-89.

[6] P. Hoffman and J.F. Humphreys, Projective Representations of the Symmetric Groups, Oxford, 1992.

[7] G. James, The Representation Theory of the Symmetric Groups, Springer Lecture Notes Math., 682 (1978).

[8] G. James and A. Kerber, The Representation Theory of the Symmetric Group, Addison-Wesley, London, 1981.

[9] I.G. Macdonald, Symmetric Functions and Hall Polynomials, 2nd edition, Oxford Univ. Press, Oxford, 1995.

[10] A.O. Morris, The spin representation of the symmetric group, Proc. London Math. Soc. (3), 12 (1962), 55-76.

[11] A.O. Morris, The spin representation of the symmetric group, Canad. J. Math., 17 (1965), 543-549.

[12] J.B. Olsson, Combinatorics and Representations of Finite Groups, Vorlesungen aus dem FB Mathematik der Univ. Essen, Heft 20, 1993.

[13] J. Stembridge, Shifted tableaux and the projective representations of symmetric groups, Adv. Math., 74 (1989), 87-134.

Received July 16, 1999 and revised October 1, 1999.

FAKULtät FÜR MATHEMATIK

Otto-von-Guericke-Universität MAgdeburG

D-39016 MagdeburG

Germany

E-mail address: bessen@mathematik.uni-magdeburg.de

Department of Mathematics

UNIVERSITY OF OREGON

Eugene, OR 97403 
E-mail address: klesh@math.uoregon.edu 